\title{
Internet visualization
}

\author{
John Heidemann • Walter Willinger
}

Published online: 12 January 2013

(C) Springer-Verlag Wien 2013

Measurement is important to understanding the Internet and related networks, and for more than a decade conferences have been dedicated to network measurement. However, in an age where increasingly large sets of semantically rich data ("big data") is being collected about today's networks, visualization plays an ever more critical role in understanding this data and ultimately the networks themselves. Yet, technical advances in network visualization often require a different perspective than is typical for network measurement.

To gauge the general interest in network visualization, provide a venue for discussing recent advances in the area, and start the process of building a bridge between the visualization and networking communities, we organized a workshop on Internet visualization (WIV 2012) that was co-located with the 2012 Internet Measurement Conference (IMC). WIV 2012 took place in the afternoon of November 13, 2012, at Boston University and was attended by 35 registered participants.

As chairs, we approached the organization of WIV 2012 somewhat experimentally in several ways. One goal was to form a technical program committee that included members from both the networking and visualization communities. A second objective was to encourage and support the use of multimedia, not just a written paper. Lastly, in addition to formal paper presentations, we wanted to provide opportunities for authors of accepted papers or extended abstracts to present their work as part of a demonstration and poster session. Although we still have a long way to go to reach the stated goals, we view WIV 2012 as a useful learning experience and first step

\footnotetext{
J. Heidemann ( $\square)$

USC/Information Sciences Institute, Marina Del Rey, CA 90292-6695, USA

e-mail: johnh@isi.edu
}

W. Willinger

AT\&T Labs-Research, Florham Park, NJ 07932, USA

e-mail: walter@ research.att.com 
at bridging the two communities, making multimedia an integral part of the paper evaluation, and growing the demo and poster session into a showcase for the latest network visualization tools and software.

We thank the ten members of the technical program committee who carefully reviewed the 11 submissions. The TPC consisted of Nevil Brownlee (University of Auckland, New Zealand, who handled the submissions that were co-authored by the co-chairs), Ramon Caceres (AT\&T Labs-Research, USA), k. Claffy (CAIDA, USA), Mark Crovella (Boston University, USA), Mike Fisk (LANL, USA), John Heidemann (co-chair, USC/ISI, USA), Stephen North (AT\&T Labs-Research, USA), Paul Rosen (University of Utah, USA), Walter Willinger (co-chair, AT\&T Labs-Research, USA), and Eiko Yoneki (University of Cambridge, UK). The TPC accepted six of the submissions for presentation at the workshop and at the demo and poster session; two additional submissions were selected for presentation at the demo and poster session only. We also thank the IMC TPC co-chairs, Ratul Mahajan (Microsoft Research) and Alex Snoeren (University of California at San Diego) for agreeing to run the demo and poster session also during IMC in an effort to provide additional exposure for this work. Finally, we want to thank the IMC general chairs, John Byers (Boston University) and Jim Kurose (University of Massachusetts Amherst), who took care of all the logistics necessary for running this successful workshop.

This special issue is a collection of all the papers that were accepted to WIV 2012, either as full papers or extended abstracts. The authors of all these papers were invited to present their work in a demo and poster session that was part of WIV 2012. The selected papers encompass topics such as information visualization (geotagged Twitter data), the use of existing Web technologies or popular data mining techniques for visualizing network traffic data, static or animated displays of sparse but large-scale Internet events, the visual discovery of correlations between different types of available network measurements, and the use of visualization for network forensics and for extracting semantics from large traceroute datasets. We believe that the papers included in this special issue are somewhat representative of the current research activities in the area of Internet visualization and hope the readers will find the contributions informative. We also invite the readers of this special issue to explore the multimedia materials associated with the papers; an on-line index is at http://www. ant.isi.edu/wiv2012/program.html with links to the authors' websites. 